\title{
Neue Herausforderungen und offene Fragen für Wissenschaft und Praxis
}

\author{
G. Richter und A. Tisch
}

Die folgende Zusammenschau soll Forschungslücken und -bedarfe zeigen sowie die Anregungen der Autorinnen und Autoren des vorliegenden Bandes zur betrieblichen und überbetrieblichen Gestaltung zusammenführen.

1. Ausgangspunkt der Überlegungen ist der von Bellmann aufgezeigte Widerspruch zwischen der volkswirtschaftlich und häufig auch erwerbsbiografisch notwendigen fortgesetzten Integration Älterer in Beschäftigung auf der einen Seite und der geringen Verbreitung betrieblicher Maßnahmen zum Erhalt der Arbeits- und Beschäftigungsfähigkeit auf der anderen Seite.

Mit dem Hinweis auf das schwache Engagement von Führungskräften und das wenig anerkannte Engagement von betrieblichen Interessenvertretungen für gesunde Arbeitsbedingungen knüpft Langhoff an dieses Umsetzungsdefizit an. Sein Blick auf relevante betriebliche Akteure lässt unterschiedliche Forschungs- und Gestaltungsperspektiven hervortreten. Über welche arbeitswissenschaftlichen und arbeitsgestalterischen Kompetenzen verfügen Führungskräfte in unterschiedlichen Branchen? Wo und wie erwerben sie praxisrelevante, aber durchaus auch theoretische Kenntnisse? Welche Strukturen unterstützen und ermutigen sie zu einer gesunden und auch alterssensiblen Führung? Welchen Stellenwert hat der Aufbau arbeitsgestalterischer Kompetenzen bei der Personalentwicklung von Führungskräften oder in Führungskräftenachwuchsprogrammen? Wie kann das Engagement der betrieblichen Interessenvertretungen für den Arbeits- und Gesundheitsschutz aufgewertet werden?

Mühlenbrock weist auf das Fehlen integrativer, verifizierter Theorien zum erfolgreichen Altern am Arbeitsplatz (,successful aging ${ }^{6}$ ) hin. Wichtige Vorarbeiten sind aktuell durch die Verknüpfung von Theorien zur Lebensspanne im Arbeitskontext mit der Handlungsregulationstheorie zu einer ,Metatheorie' (ARAL, s. Zacher, Hacker \& Frese, 2016) geleistet worden. Diese versucht zu erklären, wie Beschäftigte ihr (Arbeits-)Umfeld über die Erwerbsspanne beeinflussen und vom Umfeld beeinflusst werden. Für die Ableitung konkreter, praxisnaher Empfeh- 
lungen fehlen jedoch noch empirische Untersuchungen. Untersuchungen stehen vor dem Dilemma, entweder singuläre Merkmale (z.B. Anforderungsvielfalt) zu untersuchen und dadurch eine größere Klarheit zu Effekten im Kontext von Alter (s. Mühlenbrock \& Hüffmeier, 2020) zu liefern oder die in der betrieblichen Praxis relevanten, vielen weiteren (organisationalen) Variablen mit zu analysieren und dann wenig Kausalität zu liefern. Im Weiteren erkennt sie Forschungslücken „bei der Entwicklung von Konstrukten, die eine stärkere Vorhersagekraft als das kalendarische Alter haben und FTP (Future Time Perspective, s.o.), funktionales Alter (z.B. Gesundheit und Arbeitsfähigkeit), lebensspannenbezogenes Alter (z.B. Lebenssituation), psychosoziales Alter (z.B. soziale bzw. Selbstwahrnehmung) oder organisationales Alter (z.B. Betriebszugehörigkeit)" betrachten.

Für Richter, Kubn und Koch sollte zukünftige Forschung intersektionale Perspektiven einnehmen und mehrdimensionale Problemlagen adressieren. Wie auch Müblenbrock erkennen sie die Notwendigkeit, in zukünftigen Forschungen „das kalendarische Alter als alleinige Erklärungsvariable zu überwinden und um andere personenbezogene Variablen zu erweitern." Der Prozesscharakter des Alterns in Arbeit macht es schließlich erforderlich, lineare Modelle zu überwinden und kurvilineare Zusammenhänge in den Blick zu nehmen. Hasselhorn und Müller sprechen sich mit dem Verweis auf die Möglichkeit der Verbesserung eines schlechten Gesundheitsstatus sowie der bereits heute weitverbreiteten Praxis von Erwerbsarbeit bei schlechter Gesundheit ebenfalls für differenziertere Forschungsfragen und komplexere Forschungsdesigns aus - die nicht nur auf Gesundheit, sondern auch auf weitere individuelle Ressourcen gerichtet sein sollten. Sie fordern zudem eine umfassende Stay at Work Forschung in Deutschland. Dementsprechend sprechen sie sich für Forschung aus der Lebenslaufperspektive aus und regen ein institutionalisiertes, umfassendes, längsschnittliches „Arbeitsund Gesundheits-Monitoring" der Erwerbsbevölkerung an. Wulf zeigt betriebliche Möglichkeiten der individuellen und präventiven Arbeitsgestaltung - aber auch, dass es zur gesunden Gestaltung von Erwerbskarrieren in besonders belasteten Tätigkeiten einer besseren Verzahnung betrieblicher und überbetrieblicher Beschäftigungsmöglichkeiten (Tätigkeitswechsel) sowie eines Bewusstseins für Prävention bei den Beschäftigten bedarf.

2. Ebenfalls schon zu Beginn des Bandes arbeitet Bellmann heraus, dass Ältere, die einen Arbeitsplatz suchen, ungleich schlechtere Chancen haben, aus einem Beschäftigungsverhältnis in die Rente zu wechseln als Ältere mit einem bestehenden Arbeitsvertrag. Der Blick auf soziale Un- 
gleichheiten, die durch die Erhöhung des Renteneintrittsalters verschärft oder sogar neu entstanden sind ist ein zweiter Schwerpunkt der Beiträge. Naegele und Hess nennen als betroffene Beschäftigtengruppen „gering Qualifizierte, Frauen, Langzeitarbeitslose, gesundheitlich Beeinträchtigte, chronisch Kranke, atypisch Beschäftigte sowie ältere Arbeitnehmerinnen und Arbeitnehmer in Betrieben, in denen man aufgrund der Arbeitsbedingungen und -belastungen kaum, in Würde' bis zum gesetzlichen Renteneintrittsalter (weiter)arbeiten kann." Dabei auBer Acht gelassen scheinen noch Personen mit mehreren dieser soziodemografischen Merkmale. Brussig erkennt außerdem an, dass sich die Forschung zum Altersübergang in den zurückliegenden 20 Jahren vielfältig entwickelt hat. Allerdings ist der Kenntnisstand über Determinanten betrieblicher Strategien gegenüber unterschiedlichen Beschäftigtengruppen beim Altersübergang schwach. Brussig weist darauf hin, dass die Forschungslinie von „fallstudienbasierten Untersuchungen, die die betriebliche Personalpolitik gegenüber Älteren einbetten in die Gesamtheit von widersprüchlichen und auszubalancierenden Ansprüchen und Erwartungen von Arbeitgeberinnen und Arbeitgebern, Beschäftigten, Betriebs- und Personalräten und nicht zuletzt der Gesellschaft", weitgehend abgerissen ist. Es fehlt an aktuellen Untersuchungen, in denen Betriebe als Konfliktarena und als interessengeleitete und mit Ressourcen und strategischer Handlungsfähigkeit ausgestattete Akteure konzeptualisiert werden - insbesondere auch vor dem Hintergrund von Digitalisierung, Dekarbonisierung und Corona Pandemie.

Tophoven blickt auf bestehende gesundheitliche Ungleichheiten bei älteren erwerbstätigen Frauen. Obwohl ihr Gesundheitszustand insgesamt gut ist zeigt eine differenzierte Betrachtung, dass es auch erwerbstätige Frauen gibt, die über eine geringere funktionale körperliche Gesundheit berichten. Darüber hinaus ist zu untersuchen, ob der Weg in die Nicht-Erwerbstätigkeit (und damit Abhängigkeit) für ältere Frauen mit gesundheitlichen Einschränkungen nicht einfacher und gesellschaftlich anerkannter ist, als der möglicherweise mit vielen Hürden versehene Weg zurück in die Erwerbstätigkeit. Als Erklärungsfaktoren sind verschiedene Belastungen in der Erwerbssituation heranzuziehen, wie ein geringes Einkommen und tätigkeitbezogene Merkmale wie mangelnde Anerkennung und hohe physische Arbeitsanforderungen. Sie regt eine "holistische Perspektive“ auf die Ausgestaltung des Erwerbsverlaufs und der Erwerbssituationen von Frauen und ihren gesundheitlichen Risiken an. Die im privaten Bereich geleistete Sorgearbeit muss jedoch hinsichtlich der damit verbundenen Risiken und Chancen für gesundes Altern in Zukunft stärker in Forschungsdesigns 
einbezogen werden. Zumindest in der Forschung könnte damit ein Defizit überwunden werden, auf das Jessee, Müller und Wiegand hinweisen: Dass eine männliche Erwerbsbiografie auch für Frauen als Maßstab angesehen wird, wodurch die geschlechterspezifische Ungleichheit reproduziert wird. Deller und Naegele erkennen beim Blick auf Rentnerinnen und Rentner, die aktuell einer Beschäftigung nachgehen, „eine Dominanz geringfügiger Beschäftigungsverhältnisse, nicht unerhebliche Geschlechter- und Bildungsunterschiede, einen starken Gesundheitsbias und oftmals wenig Qualifizierungs- und Karriereentwicklungsangebote." Auch ist von Interesse, inwieweit die „neue“ Beschäftigtengruppe der erwerbstätigen Rentnerinnen und Rentner tradierte Handlungsweisen und Karrierewege in der betrieblichen Praxis verändern und wie sie sich insgesamt in existierende Betriebsgefüge eingliedern. Weitere Forschung sollte „ein Augenmerk auf die Gefahr der Verfestigung sozialer Ungleichheiten, die im Zuge des Ausbaus von Erwerbstätigkeit im Rentenalter entstehen können, (zu) werfen. Dazu müssen Wege und Maßnahmen entwickelt und validiert werden, welche die individuellen, organisationalen und gesellschaftlichen Chancen - die in verlängerten Erwerbskarrieren stecken - fördern, ohne dabei bestimmte (prekäre) Beschäftigungsgruppen zu exkludieren."

3. Bei der betrieblichen Gestaltung des gesunden Alterns in Arbeit steht bislang die Personalarbeit im Vordergrund. Reindl bilanziert, dass viele innovative Konzepte und Tools entwickelt wurden, dass aber das Handlungsprogramm des gesunden und produktiven Alterns oft Stückwerk oder Insellösung geblieben ist. Ein wichtiges Instrument ist dabei das Mitarbeitergespräch als Grundlage der Personalentwicklung - und damit auch ein wichtiger Gegenstand zukünftiger Forschungen. Diese sollten jedoch nicht auf die operative Ebene beschränkt bleiben, sondern auch strategische Fragestellungen adressieren. Für Guggemos und Conrads wird ,aus wissenschaftlicher Sicht deutlich, dass betriebsindividuelles Lernen über mehrere Stufen hin zu einem integrierten lernenden System wünschenswert wäre und prozessualer Unterstützung inklusive einer Informations- und Weiterbildungsstrategie bedarf. Der Unterstützungsbedarf gilt umso mehr, je kleiner die Unternehmen und je geringer die quantitativen und fachlichen Personalmanagement-Ressourcen im jeweiligen Betrieb sind. Besonderes Augenmerk benötigen Tätigkeitsbereiche, die mit hohen Arbeitsbelastungen und zugleich niedrigen qualifikatorischen Voraussetzungen einhergehen. Von Relevanz ist dies insbesondere dann, wenn die jeweilige Branche eher wenig lukrativ ist, und deshalb auch nur wenig finanzielle Mittel für Personalmaßnahmen zur Verfügung stehen." Naegele und Hess weisen da- 
rauf hin, dass sich in Deutschland bisher nur wenige Kosten-NutzenAnalysen von förderlichen Maßnahmen der Verlängerung von Lebensarbeitszeit und darauf bezogener "Good practices“ finden. Diese sollten in Zukunft in Modellprojekten und Forschungsvorhaben verstärkt erarbeitet werden. Kast wie auch Greiff und Krüger sehen in der zunehmenden Diversität der Belegschaften Herausforderungen für Personalarbeit, Führung und Arbeitsgestaltung - und auch Forschungsthemen von hoher betrieblicher Relevanz.

Decius betont ebenfalls den großen Stellenwert des kontinuierlichen Lernens in der Erwerbsbiografie. Er lenkt die Aufmerksamkeit auf das informelle Lernen und das Job-Crafting-Verhalten. „Die Hebel zur Förderung des informellen Lernens und des Job-Crafting-Verhaltens bedürfen weiterer Forschungsbemühungen. Insbesondere sollte der Frage nachgegangen werden, wie sich informelles Lernen am Arbeitsplatz fördern lässt und auf welche Weise insbesondere ältere Beschäftigte zu Job-Crafting-Verhalten motiviert werden können.“ Antworten sollten praktische Empfehlungen und Werkzeuge enthalten, um lernförderliche Rahmenbedingungen zu schaffen und die Eigenverantwortung der Beschäftigten zu stärken.

4. Overhage weitet den Blick und fragt: „Greift das Konzept einer alter(n)sgerechten Gestaltung der Arbeit noch angesichts agiler Arbeitsformen?" Er betont, dass Motivation und soziale Dimensionen zentrale Faktoren sind, damit Ältere die im Zuge der Digitalisierung wachsenden Freiräume konstruktiv nutzen: „Denn auch in Zukunft wird nach Prof. Juhani Ilmarinen das, Wollen, Können und Dürfen' entscheidend sein." Für Schaper dürfen Tätigkeitsanforderungen und der Autonomiegrad auf der einen Seite nicht zu groß werden, da diese ansonsten zu einer Überforderung der Personen führen und Lernprozesse eingeschränkt bzw. behindert werden. Auf der anderen Seite erfordert arbeitsintegriertes Lernen motivationale Unterstützung. Forschungen zur lernförderlichen Arbeitsgestaltung sollte sich auf „die Rolle metakognitiver (z.B. in Form bestimmter Lernstrategien und Lernformen) und behavioraler Prozesse (z.B. in Form von Explorationsverhalten)" konzentrieren. Ein weiteres Forschungs- und Interventionsfeld, das auch durch die sprunghaft gestiegene Verbreitung von Home Office, aber auch durch die zunehmende Nutzung von E-Learning im betrieblichen Kontext an Relevanz gewinnt, sind selbstgesteuertes Lernen und selbstorganisierte Kompetenzentwicklung.

5. Naegele und Hess unterstreichen, dass zum gesunden Altern in Erwerbsarbeit auch Würde, also soziale Anerkennung und Selbstachtung gehört. Bode und Krassen sehen ebenfalls Forschungsbedarf bei der Bewäl- 
tigung der Herausforderungen im Kontext des digitalen und demografischen Wandels. „Bei der Einführung von KI in die Arbeitswelt ist es wichtig, Chancen und Risiken für das jeweilige Unternehmen und seine Beschäftigten strukturiert, spezifisch und zielgerichtet zu berücksichtigen. Die Forschung in diesem Feld muss Möglichkeiten eröffnen, die als gute Beispiele von den Unternehmen als Prototypen für eigene Entwicklungen genutzt werden können." Fassio, Schmeink und Zeckra weisen in diesem Zusammenhang auf das Potenzial von Unternehmensnetzwerken für die Lösung offener Fragen hin.

6. Damit gesundes Altern in Arbeit bei erhöhten Altersgrenzen und verändertem Erwerbsverhalten für alle Beschäftigten verwirklicht werden kann, müssen Institutionen und Akteure verschiedener gesellschaftlicher Teilbereiche koordiniert werden und zuverlässig und pragmatisch zusammenarbeiten. Für Richter, Kubn und Koch fehlt heute wie vor 20 Jahren eine abgestimmte und dem Erhalt der Arbeitsfähigkeit verpflichtete Unterstützungsinfrastruktur für Kleinbetriebe. „Sozialversicherungen, Kammern, kommunale Wirtschaftsförderung, Gewerbeaufsicht, Arbeitsagenturen und Job-Center bearbeiten Teilaspekte und folgen jeweils unterschiedlichen institutionellen Logiken." Forschung zur Entwicklung von Regelungen, die systemübergreifend wirken, steht damit auf der Tagesordnung. Dabei sollte das Präventionsgesetz einbezogen werden. Naegele und Hess fordern Mehr-Ebenen-Analysen, auch „damit „Frühwarnsysteme“ wie Risiko- oder Gefährdungsanalysen verwirklicht werden können“, um Gesundheits- oder Qualifikationsproblemen vorzubeugen. Deller und Naegele halten für Analysen der zunehmenden Erwerbstätigkeit im Ruhestand ebenfalls Mehrebenenbetrachtungen für erforderlich, um das Zusammenspiel von Makro- (z.B. politische und gesellschaftliche Randbedingungen etc.), Meso- (organisationale, institutionelle und private, außerberufliche Stellgrößen etc.) und Mikrofaktoren (Motivationsdimensionen, Persönlichkeitsmerkmale, Zielorientierungen etc.) angemessen untersuchen zu können. „Dies erfordert transdisziplinäre Forschungsgruppen, entsprechend gestaltete innovative Datenerhebungen und Weiterentwicklungen in der Modellierung."

7. Anlauft und Feldes arbeiten Erfahrungen mit „Tarifverträgen Demografie" im Bereich der IG Metall auf. Sie fordern zum einen mehr Verbindlichkeit und ggfs. Sanktionen, wenn Umsetzungsdefizite im Bereich des Arbeits- und Gesundheitsschutzes und Regelverstöße gegen diese Vereinbarungen festgestellt werden. Zum anderen regen sie die Gründung von paritätisch besetzten „Agenturen für gute Transformation" an, die Überwachungs-, Entwicklungs- und Kommunikationsaufga- 
ben übernehmen und aus einem Tariffonds bzw. einem Mindestanteil der Lohn- und Gehaltssumme finanziert werden. Als Gegenstand zukünftiger Forschungsarbeiten schlagen Anlauft und Feldes den Zusammenhang von digitaler Transformation und demografischen Veränderungen mit dem Ziel, in kurzer Zeit mehr Handlungs- und Gestaltungssicherheiten für eine zukunftsfeste Weiterbildung und für neue Impulse bei der Gestaltung lernförderlicher und gesundheitsverträglicher Arbeit zu gewinnen, vor. Erwünscht sind auch „Detailerkenntnisse zur Subjektseite technologischer Veränderungen, die folgende Forschungsfragen beantworten können: ,Wie können Transformationsprozesse so initiiert und gesteuert werden, dass sie den unterschiedlichen Bedürfnissen und Kompetenzen der Generationen und Berufsgruppen gerecht werden? Worauf haben die verantwortlichen Prozessowner zu achten, damit der Fachlichkeit, dem Entdeckergeist, dem Produzentenstolz und den Lebensleistungen der einzelnen Beschäftigten gebührend Rechnung getragen wird?' Es bedarf fundierter Analysen, die sowohl bestehende Defizite wie auch die Entwicklungschancen und -risiken der Transformationsvorgänge kenntlich machen. Es ist notwendig, mehr zu erfahren über Widersprüche, Konfliktpotenziale und systemische Wechselwirkungen zwischen unterschiedlichen Dimensionen des Wandels. Systematisch zu durchleuchten wäre ferner das Wirkungsverhalten von Vereinbarungsinstrumenten und Personalstrategien."

8. Eine weitere häufig thematisierte Herausforderung besteht im verbesserten Austausch von Wissenschaft und Arbeitswelt. Falkenstein und Kardys fordern eine beharrliche Wissensvermittlung zum Thema Alter und Arbeit in den Betrieben und vor politischen Gremien. Langhoff und Müblenbrock fordern Forschungen zur Überwindung des „Knowing-Doing-Gap“: Was behindert z.B. eine Umsetzung alternsgerechter Arbeitsgestaltung in der Praxis? Hasselhorn und Müller fordern von der Politik, Forschungen zu initiieren, die nicht darauf schauen, wann die Älteren aus dem Erwerbsleben aussteigen, sondern wie sich die letzten Erwerbsjahre (und die folgenden Jahre in Rente) für sie darstellen. Gebhardt und Linner berichten von internationalen Diskussionen im Rahmen von ISO mit dem Ziel, Empfehlungen für eine „age-inclusive workforce“ zu geben, die für möglichst viele Organisationen anwendbar sind. 
